\title{
PROJECTIONS AND APPROXIMATE IDENTITIES FOR IDEALS IN GROUP ALGEBRAS
}

\author{
BY
}

\author{
TENG-SUN LIU( ${ }^{1}$, ARNOUD VAN ROOIJ AND JU-KWEI WANG
}

ABSTRACT. For a locally compact group $G$ with property $\left(\mathrm{P}_{1}\right)$, if there is a continuous projection of $L^{1}(G)$ onto a closed left ideal $I$, then there is a bounded right approximate identity in $I$. If $I$ is further 2 -sided, then $I$ has a 2 sided approximate identity. The converse is proved for $w^{*}$-closed left ideals.

Let $G$ be further abelian and let $I$ be a closed ideal in $L^{1}(G)$. The condition that $I$ has a bounded approximate identity is characterized in a number of ways which include (1) the factorability of $I,(2)$ that the hull of $I$ is in the discrete coset ring of the dual group, and (3) that $I$ is the kernel of a closed element in the discrete coset ring of the dual group.

Introduction. Let $G$ be a locally compact group, $I$ a closed left ideal in $L^{1}(G)$ and $P$ a continuous projection of $L^{1}(G)$ onto $I$. It is proved by $W$. Rudin [11, Theorem 1] that, if $G$ is compact, there exists a continuous projection $Q$ of $L^{1}(G)$ onto $I$ such that

$$
f * Q g=Q(f * g) \quad\left(f, g \in L^{1}(G)\right) .
$$

Further [11, Proof of Theorem 2], if in addition $G$ is abelian, then there exists an idempotent measure $\mu$ on $G$ such that

$$
Q f=f * \mu \quad\left(f \in L^{1}(G)\right)
$$

so that $Q$ is actually an algebra homomorphism. It follows that $I$, considered as a Banach algebra, has a bounded approximate identity.

The purpose of Part I of this paper is to find out what happens if $G$ is not compact or abelian. It turns out that if $G$ has the property $\left(\mathrm{P}_{1}\right)$ (which it does if it is compact) then the projection $P$ leads to a net of projections $Q$ for which the formula (*) "almost" holds, and that $I$ still has a bounded (right) approximate identity (Theorem 2).

Received by the editors December 10, 1971.

AMS (MOS) subject classifications (1970). Primary 22B 10, 22D15, 43A20; Secondary 43A45, 46H10.

Key words and phrases. Continuous projections, bounded approximate identities, closed left ideal s, closed right ideals, closed 2-sided ideals, group algebras, modular functions, property $\mathrm{P}_{1}, w^{*}$-closed, Calderón set, spectral set, discrete coset ring, factorable closed ideals, Bohr compactification.

(1) Research supported in part by the National Science Foundation. An announcement of the results of this paper has been published in C. R. Acad. Sci. Paris 272 (1971), 473. 
The converse problem (If $I$ is a closed left ideal in $L^{1}(G)$ that has a bounded right approximate identity, does there necessarily exist a continuous projection of $L^{1}(G)$ onto $I$ ?) seems still to be open. Under an additional condition on $I$ the question can be answered in the affirmative (Theorem 4).

Next we turn to the case where $G$ is abelian. Let $\Gamma$ be its dual group. Let $X$ be a closed subset of $\Gamma$, and $J$ a closed translation invariant linear subspace of $L^{\infty}(G)$ so that $X=\Gamma \cap J$. J. E. Gilbert [4], basing himself upon H. P.

Rosenthal's paper [9], proves that there exists a continuous projection of $L^{\infty}(G)$ onto $J$ if and only if $X$ is an element of the coset ring of the discrete group $\Gamma_{d}$ that has the same underlying group as $\Gamma$. Furthermore, he proves that then $X$ must be what W. Rudin [12] calls a " $C$-set". Thus he brings about a connection between projections and approximate identities. (See also [11], [3],[10].) His most important tool is a theorem that describes all closed subsets of $\Gamma$ that lie in the coset ring of $\Gamma_{d}$. (See also [13].)

In Part II we continue Gilbert's investigations, and characterize the closed ideals in $L^{1}(G)$ that have bounded approximate identities. At the same time we prove that the se ideals are just the ideals that are factorable. (A commutative Banach algebra $A$ is called factorable if there is a $c>0$ such that for every $a \in A$ and $\epsilon>0$ there exist $x, y \in A$ for which $a=x y,\|x\|<c$ and $\|a-y\|<\epsilon$.) This partly solves a problem raised by Hewitt and Ross $[6, \$ 39.40]$.

Preliminaries. For most notations we follow the conventions used by [6] and [12]. We use, however, the multiplicative notation even in the abelian case.

Let $G$ be a locally compact group. By $C_{0}(G)$ we denote the Banach space of all continuous functions $G \rightarrow \mathrm{C}$ that vanish at infinity. The dual space, $C_{0}(G)^{*}$, of $C_{0}(G)$ is identified with the Banach space $M(G)$ of all bounded Radon measures on $G . M(G)$ is a Banach algebra under convolution. The $w^{*}$-topology on $M(G)$ is the weak topology determined by $C_{0}(G)$.

We select a left Haar measure on $G$. The Haar integral of a function $f$ : $G \rightarrow \mathrm{C}$ is written $\int f(x) d x$. The Banach space $L^{1}(G)$ of equivalence classes of integrable functions is identified in the usual way with a subspace of $M(G)$. Thus, $L^{1}(G)$ is a two-sided ideal in $M(G)$.

Deviating from the notation of [6], for $f \in L^{1}(G)$ and $x \in G$ we define

$$
\begin{aligned}
& f_{x}(y)=f(x y), \quad f^{x}(y)=f(y x) \Delta(x) \quad(y \in G), \\
& f^{\prime}(y)=f\left(y^{-1}\right) \Delta\left(y^{-1}\right) \quad(y \in G),
\end{aligned}
$$

where $\Delta$ is the modular function of $G$. Then $f_{x}, f^{x}, f^{\prime} \in L^{1}(G)$ and $\left\|f_{x}\right\|=\left\|f^{x}\right\|=$ $\left\|f^{\prime}\right\|=\|f\|$. One easily establishes the relations

$$
(f * g)_{x}=f_{x} * g, \quad(f * g)^{x}=f * f^{x}, \quad f * g_{x}=f^{x} * g, \quad(f * g)^{\prime}=g^{\prime} * f^{\prime} .
$$

Further, 


$$
(f * g, b)=\left(g, f^{\prime} * b\right) \quad\left(f, g \in L^{1}(G) ; b \in L^{\infty}(G)\right) .
$$

For every $f \in L^{1}(G)$ the formulas

$$
x \mapsto f_{x} \text { and } x \mapsto f^{x}
$$

define bounded continuous maps $G \rightarrow L^{1}(G)$. Using the terminology of vectorvalued integration [1] one obtains

$$
f * g=\int f(x) g_{x-1} d x \quad\left(f, g \in L^{1}(G)\right) .
$$

Similarly, for $j \in L^{\infty}(G)$ and $x \in G$ put $j_{x}(y)=j(x y)(y \in G)$. For $j \in C_{0}(G)$, $x \mapsto j_{x}$ is a continuous map $G \rightarrow C_{0}(G)$, and

$$
f * j=\int f(x) j_{x^{-1}} d x \quad\left(f \in L^{1}(G) ; j \in C_{0}(G)\right) .
$$

$G$ is said to have the property $\left(\mathrm{P}_{1}\right)$ if for all compact sets $C \subset G$ and $\epsilon>0$ there exists an $b \in L^{1}(G), b \geq 0,\|b\|=1$, such that $\left\|b_{x}-b\right\| \leq \epsilon$ for all $x \in C$ (see [7, Chapter 8]). Equivalently, for all compact $C \subset G$ and $\epsilon>0$ there exists an $b \in L^{1}(G), b \geq 0,\|b\|=1$, such that $\left\|b^{x}-b\right\| \leq \epsilon$ for all $x \in C$. If $G$ has the property $\left(\mathrm{P}_{1}\right)$, then there exists a left invariant mean on $L^{\infty}(G)$ (i.e., an $M \epsilon$ $L^{\infty}(G)^{*}$ such that $\|M\|=1, M 1=1$ and $M j_{x}=M j$ for all $j \in L^{\infty}(G)$ and $\left.x \in G\right)$. For the proof of this statement, see [7, Chapter $8, \S 6]$ and $[5]$, where it is also proved that the existence of a left invariant mean implies the property $\left(P_{1}\right)$. All compact groups and all abelian locally compact groups have the property $\left(\mathrm{P}_{1}\right)$.

Part I. Projections onto ideals of group algebras. Our $f$ irst lemma is a direct descendant of $[11$, Theorem 1$]$. By $\mathcal{P}^{\circ}\left(L^{1}(G)\right)$ we denote the space of all continuous linear maps $L^{1}(G) \rightarrow L^{1}(G)$.

Lemma 1. Let $G$ be a locally compact group. For every $T \in \varrho^{\rho}\left(L^{1}(G)\right)$ and $b \in L^{1}(G)$ the formula

$$
T_{b} f=\int b(x)\left(T\left(f_{x}\right)\right)_{x-1} d x \quad\left(f \in L^{1}(G)\right)
$$

defines $a T_{b} \in \mathfrak{L}\left(L^{1}(G)\right)$ for which $\left\|T_{b}\right\| \leq\|b\|\|T\|$ and

(ii) $\left\|f * T_{b} g-T_{b}(f * g)\right\| \leq \int|f(x)|\left\|b_{x}-b\right\| d x \cdot\|T\|\|g\| \quad\left(f, g \in L^{1}(G)\right)$.

If $G$ bas the property $\left(\mathrm{P}_{1}\right)$, then for all $f_{0}, f_{1}, \cdots, f_{n} \in L^{1}(G)$ and $\epsilon>0$ there exists an $b \in L^{1}(G), b \geq 0,\|b\|=1$, sucb that for each $i$,

$$
\left\|f_{i} * T_{b} g-T_{b}\left(f_{i} * g\right)\right\| \leq \epsilon\|T\|\|g\| \quad\left(g \in L^{1}(G) ; T \in \mathcal{I}\left(L^{1}(G)\right) .\right.
$$

Proof. If $f \in L^{1}(G)$ then $x \mapsto\left(T f_{x}\right)_{x-1}$ is a continuous map $G \rightarrow L^{1}(G)$ $\left[6\right.$, Theorem 20.4] and $\left\|\left(T f_{x}\right)_{x-1}\right\| \leq\|T\|\|f\|$ for all $x$. It follows [1, 1 , Proposition 81 that the integral in (i) defines $T_{b} \in \mathcal{\perp}\left(L^{1}(G)\right)$ and that $\left\|T_{b}\right\| \leq\|b\|\|T\|$. 
Take $g \in L^{1}(G)$. For every $a \in G$,

$$
\begin{aligned}
T_{b}\left(g_{a^{-1}}\right) & =\int b(x)\left(T g_{a^{-1} x_{x-1}}\right) d x=\int b(a x)\left(T g_{x}\right)_{x^{-1} a^{-1}} d x \\
& =\left[\int b_{a}(x)\left(T g_{x}\right)_{x^{-1}} d x\right]_{a^{-1}}=\left(T_{b_{a}} g_{a^{-1}} .\right.
\end{aligned}
$$

Then for all $f \in L^{1}(\bar{G})$,

$$
\begin{aligned}
f * T_{b} g & -T_{b}(f * g)=\int f(x)\left(T_{b} g\right)_{x^{-1}} d x-T_{b}\left(\int f(x)_{x^{-1}} d x\right) \\
& =\int f(x)\left(T_{b} g\right)_{x^{-1}} d x-\int f(x) T_{b}\left(g_{x^{-1}}\right) d x \\
& =\int f(x)\left(T_{b} g-T_{b_{x}} g\right)_{x-1} d x=\int f(x)\left(T_{b-b_{x}}(g)\right)_{x^{-1}} d x .
\end{aligned}
$$

(ii) follows, since $\left\|\left(T_{b-b_{x}}(g)\right)_{x-1}\right\|=\left\|T_{b-b_{x}}(g)\right\| \leq\left\|b-b_{x}\right\|\|T\|\|g\|$.

Now assume that $G$ has the property $\left(\mathrm{P}_{1}\right)$. Let $f_{0}, \cdots, f_{n} \in L^{1}(G)$ and $\epsilon>0$. We may assume $\left\|f_{i}\right\| \leq 1$ for each $i$. Take a compact set $C \subset G$ such that $\int_{G \backslash C}\left|f_{i}\right| \leq 1 / 4 \epsilon$ for each $i$, and an $b \in L^{1}(G), b \geq 0,\|b\|=1$ such that $\left\|b-b_{x}\right\| \leq 1 / 4 \epsilon$ for all $x \in C$. For every $f_{i}$,

$$
\int_{C}\left|f_{i}(x)\right|\left\|b-b_{x}\right\| d x \leq \int_{G}\left|f_{i}(x)\right| d x \cdot 1 / 4 \epsilon \leq 1 / 4 \epsilon
$$

and

$$
\int_{G \backslash C}\left|f_{i}(x)\right|\left\|b-b_{x}\right\| d x \leq \int_{G \backslash C}\left|f_{i}(x)\right| d x \cdot 2\|b\| \leq 1 / 2 \epsilon
$$

(iii) follows.

Let $A$ be a closed subalgebra of $L^{1}(G)$. A net $\left(u_{i}\right)$ in $A$ is a left approximate identity in $A$ if $\lim u_{i} * f=f$ for all $f \in A$. It is called bounded if sup $\left\|u_{i}\right\|<\infty$. A moment's reflection shows that $A$ has a bounded left approximate identity if and only if there is a $c>0$ such that for any $f_{1}, \cdots, f_{n} \in A$ and $\epsilon>0$ there is a $u \in A$ such that $\|u\| \leq c$ and $\left\|u * f_{i}-f_{i}\right\| \leq \epsilon \quad(i=1,2, \cdots, n)$. Right and two-sided approximate identities are defined analogously. A linear map $T$ : $L^{1}(G) \rightarrow A$ that leaves every element of $A$ fixed is a projection of $L^{1}(G)$ onto $A$.

Theorem 2. Let $G$ be a locally compact group that bas the property $\left(\mathrm{P}_{1}\right)$. Let $I$ be a closed left [ right; two-sided] ideal in $L^{1}(G)$, such that there exists a continuous projection $P$ of $L^{1}(G)$ onto $I$. Then $I$ bas a right [left; two-sided] approximate identity of bound $\leq\|P\|$.

Proof. Assume that $I$ is a closed left ideal. For every $f \in L^{1}(G)$ we have $\left(P f_{x}\right)_{x-1} \in I(x \in G)$; thus, every $P_{b}$ maps $L^{1}(G)$ into $I$. Further, if $f \in I$, then for every $x$ we have $f_{x} \in I$, so $\left(P f_{x}\right)_{x-1}=f_{x x^{-1}}=f$. Hence, if $\int b=1$, then $P_{b}$ is a projection of $L^{1}(G)$ onto $I$.

Now take $f_{1}, \cdots, f_{n} \in I, \epsilon>0$. By Lemma 1 (iii), and the above there exists a projection $Q$ of $L^{1}(G)$ onto $I$ such that $\|Q\| \leq\|P\|$ while for each $i$, 


$$
\left\|f_{i} * g g-Q\left(f_{i} * g\right)\right\| \leq \epsilon\|P\|^{-1}\|Q\|\|g\| \leq \epsilon\|g\| \quad\left(g \in L^{1}(G)\right) .
$$

Choose $g \in L^{1}(G)$ so that $\|g\|=1$ while $\left\|f_{i} * g-f_{i}\right\| \leq \epsilon\|Q\|^{-1}$ for each $i$. Then $Q g \in I,\|Q g\|<\|P\|$, and

$$
\left\|f_{i} * Q g-f_{i}\right\| \leq\left\|f_{i} * Q g-Q\left(f_{i} * g\right)\right\|+\left\|Q\left(f_{i} * g\right)-Q f_{i}\right\| \leq 2 \epsilon \quad(i=1, \cdots, n) .
$$

In a similar way one can attack the right ideal. For two-sided ideals we prove, somewhat more generally

Theorem 3. Let $G$ be a locally compact group with the property $\left(\mathrm{P}_{1}\right)$. Let I be a closed two-sided ideal in $L^{1}(G)$ that bas a left approximate identity of bound c. Then I has a two-sided approximate identity of bound $c$.

Proof. Take $f_{1}, \cdots, f_{n} \in I, \epsilon>0$. We construct a $w \in I,\|w\| \leq c$, such that for each $i,\left\|f_{i} * w-f_{i}\right\| \leq 3 c \epsilon+2 \epsilon$ and $\left\|w * f_{i}-f_{i}\right\| \leq 2 c \epsilon+2 \epsilon$.

Take $f_{0} \in L^{1}(G),\left\|f_{0}\right\| \leq 1$ so that $\left\|f_{i} * f_{0}-f_{i}\right\| \leq \epsilon$ and $\left\|f_{0} * f_{i}-f_{i}\right\| \leq \epsilon$ $(i=1, \cdots, n)$. Let $b$ be as in Lemma 1(iii). Take a compact $C \subset G$ such that $\int_{G \backslash C}|b| \leq \epsilon$. There exist $x_{1}, \cdots, x_{m} \in C$ such that for each $i$ and every $x \in C$ there is an $x_{j}$ with $\left\|\left(f_{i}\right)_{x_{j}}-\left(f_{i}\right)_{x}\right\| \leq 1 / 2(1+c)^{-1} \epsilon$. By our assumption on $I$ we can find a $u \in I,\|u\| \leq c$, such that

$$
\left\|\dot{u} *\left(f_{i}\right)_{x_{j}}-\left(f_{i}\right)_{x_{j}}\right\| \leq 1 / 2 \epsilon \text { for all } i, j .
$$

Then

$$
\left\|u *\left(f_{i}\right)_{x}-\left(f_{i}\right)_{x}\right\| \leq \epsilon \quad(x \in C ; i=1, \cdots, n) .
$$

Now put $T f=u * f\left(f \in L^{1}(G)\right)$. From Lemma 1 we obta in

$$
\left\|f_{i} * T_{b} g-T_{b}\left(f_{i} * g\right)\right\| \leq \epsilon c\|g\| \quad\left(g \in L^{1}(G) ; i=0, \cdots, n\right) .
$$

Further, for every $f \in L^{1}(G)$,

so that

$$
\begin{aligned}
T_{b} f-f & =\int h(x)\left[\left(u * f_{x}\right)_{x-1}-f\right] d x=\int b(x)\left[u * f_{x}-f_{x}\right]_{x-1} d x \\
& =\int_{C} b(x)\left[u * f_{x}-f_{x}\right]_{x-1} d x+\int_{G \backslash C} b(x)\left[u * f_{x}-f_{x}\right]_{x-1} d x
\end{aligned}
$$

$$
\left\|T_{b} f_{i}-f_{i}\right\| \leq \epsilon(c+2) \quad(i=0, \cdots, n) .
$$

Finally, observe that $x \mapsto u_{x-1}^{x}$ is a bounded continuous map of $G$ into $I$, so that $\int b(x) u_{x-1}^{x} d x$ is an element of $I$. For all $f \in L^{1}(G)$,

$$
\begin{aligned}
T_{b} f & =\int h(x)\left(u * f_{x}\right)_{x-1} d x=\int b(x)\left(u_{x-1} * f_{x}\right) d x \\
& =\int b(x)\left(u_{x-1}^{x} * f\right) d x=\left(\int b(x) u_{x-1}^{x} d x\right) * f .
\end{aligned}
$$

Consequently, $T_{b}$ maps $L^{1}(G)$ into $I$, and

$$
\left(T_{b} f\right) * g=T_{b}(f * g) \quad\left(f, g \in L^{1}(G)\right) .
$$


Now put $w=T_{b} f_{0}$. Then $w \in I,\|w\| \leq\|T\|\|b\|\left\|f_{0}\right\| \leq\|u\| \leq c$; and by applying (i), (ii) and (iii) above, we see that for $i=1, \cdots, n$,

$$
\begin{aligned}
\left\|f_{i} * w-f_{i}\right\| & \leq\left\|f_{i} * T_{b} f_{0}-T_{b}\left(f_{i} * f_{0}\right)\right\|+\left\|T_{b}\left(f_{i} * f_{0}-f_{i}\right)\right\|+\left\|T_{b} f_{i}-f_{i}\right\| \\
& \leq \epsilon c\left\|f_{0}\right\|+\|T\| \epsilon+\epsilon(c+2) \leq \epsilon(3 c+2)
\end{aligned}
$$

and

$$
\begin{aligned}
\left\|w * f_{i}-f_{i}\right\|=\left\|T_{b}\left(f_{0} * f_{i}\right)-f_{i}\right\| & \leq\left\|T_{b}\left(f_{0} * f_{i}-f_{i}\right)\right\|+\left\|T_{b} f_{i}-f_{i}\right\| \\
& \leq\|T\| \epsilon+\epsilon(c+2) \leq \epsilon(2 c+2) .
\end{aligned}
$$

Remarks. A closed left ideal $I$ in $L^{1}(G)$ for which there exists a continuous projection of $L^{1}(G)$ onto $I$ may fail to possess a left approximate identity-even an unbounded one. A sufficiently weird example seems to be the following. Let $G$ be the group generated by two elements, $a$ and $b$, with the relations $a^{2}=b^{3}=1$, $a=b a b\left(G\right.$ is isomorphic to $S_{3}$ ). Consider the functions

$$
\begin{aligned}
& f=1 / 2 \chi\{1\}+1 / 2 \chi\{a\}-1 / 6, \\
& g=1 / 2 \chi_{\left\{b^{2}\right\}}+1 / 2 \chi\{a b\}-1 / 2 \chi\{b\}-1 / 2 X\{b a\}
\end{aligned}
$$

where $\chi$ denotes characteristic function. The two-dimensional subspace $I$ of $L^{1}(G)$ generated by $f$ and $g$ is a left ideal; obviously there is a continuous projection of $L^{1}(G)$ onto $I$. For $f$ and $g$ we have the relations

$$
f * f=f, f * g=0, g * f=f, g * g=0 .
$$

It follow that $f$ is a right unit, but $I$ does not have a left approximate identity.

If $G$ does not have the property $\left(\mathrm{P}_{1}\right)$, then $I^{1}(G)=\left\{f \in L^{1}(G): \int f=0\right\}$ is a two-sided ideal such that there is a continuous projection of $L^{1}(G)$ onto $I^{1}(G)$, while $I^{1}(G)$ does not have a (bounded or unbounded) right approximate identity. (See [8, Proof of Theorem II]. Note that Reiter's definition of a bounded right approximate identity is different from ours.)

Now we view $L^{1}(G)$ as a subspace of $M(G)$. The weak topology of $L^{1}(G)$, induced by $C_{0}(G)$, will be called the $w^{*}$-topology. It is now not hard to prove a converse to Theorem 2 for $w^{*}$-closed ideals.

Theorem 4. Let $G$ be a locally compact group with the property $\left(\mathrm{P}_{1}\right)$. Let I be a $w^{*}$-closed left ideal in $L^{1}(G)$ and $I^{\perp}=\left\{b \in L^{\infty}(G):(f, b)=0\right.$ for all $\left.f \in I\right\}$. The following conditions are equivalent:

(a) I has a bounded right approximate identity.

( $\beta$ ) There exists an idempotent $\mu \in M(G)$ such that $I=L^{1}(G) * \mu$.

(y) There exists a continuous projection $P$ of $L^{1}(G)$ onto $I$.

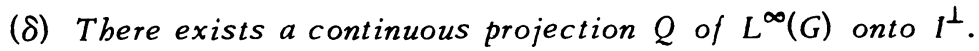

Proof. We prove the implications $(\alpha) \Rightarrow(\delta) \Rightarrow(\beta) \Rightarrow(\gamma)$; the implication $(y) \Longrightarrow(\alpha)$ is contained in Theorem 2 . 
$(a) \Rightarrow(\delta)$. Let $\Omega$ denote the natural map $L^{1}(G) \rightarrow L^{\infty}(G)^{*}$. Let $\left(u_{i}\right)$ be a bounded right approximate identity in $I$. By the Alaoglu theorem the net $\left(\Omega u_{i}\right)$ has a $w^{*}$-convergent cofinal subnet; we may assume that it is itself $w^{*}$-convergent in $L^{\infty}(G)^{*}$. Then for every $b \in L^{\infty}(G), \lim \left(u_{i}, b\right)$ exists. The formula

$$
(f, Q b)=(f, b)-\lim \left(u_{i}, f^{\prime} * b\right) \quad\left(f \in L^{1}(G) ; b \in L^{\infty}(G)\right)
$$

defines a continuous linear map $Q: L^{\infty}(G) \rightarrow L^{\infty}(G)$. If $f \in I$, then for all $h$, $(f, Q b)=(f, b)-\lim \left(f * u_{i}, b\right)=0$; so $Q$ maps $L^{\infty}(G)$ into $I^{\perp}$. Conversely, for any $b \in I^{\perp}$ and $f \in L^{1}(G),(f, Q b-b)=\lim \left(u_{i}, f^{\prime} * b\right)=\lim \left(f * u_{i}, b\right)=0$; so $Q b=b$. Hence $Q$ is a projection of $L^{\infty}(G)$ onto $I^{\perp}$.

$(\delta) \Rightarrow(\beta)$. As $G$ has the property $\left(\mathrm{P}_{1}\right)$ there exists a translation invariant mean $M$ on the space of all bounded continuous functions on $G$. Instead of $M b$ we shall write $M_{x} b(x)$. Define a continuous linear $R: C_{0}(G) \rightarrow L^{\infty}(G)$ by

$$
(f, R j)=M_{x}\left(f_{x}, Q\left(j_{x}\right)\right) \quad\left(f \in L^{1}(G) ; j \in C_{0}(G)\right) .
$$

For all $a \in G,\left(f,(R j)_{a}\right)=\left(f_{a^{-1}}, R j\right)=M_{x}\left(f_{a^{-1} x_{x}}, Q j_{x}\right)=M_{x}\left(f_{x}, Q j_{a x}\right)=\left(f, R\left(j_{a}\right)\right)$. Hence, $(R j)_{a}=R\left(j_{a}\right)$ for all $j \in C_{0}(G)$ and $a \in G$. It follows that, for all $g \in L^{1}(G)$ and $j \in C_{0}(G)$,

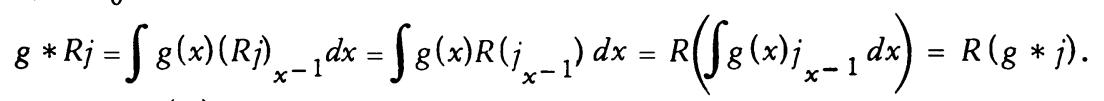

Define $\mu \in M(G)$ by

$$
(\mu, j)=j(1)-R j(1) \quad\left(j \in C_{0}(G)\right) .
$$

If $f \in L^{1}(G)$ and $j \in C_{0}(G)$, then $(f * \mu, j)=\left(\mu, f^{\prime} * j\right)=\left(f^{\prime} * j\right)(1)-\left(f^{\prime} * R j\right)(1)=$ $(f, j-R j)$. Therefore, $(f * \mu, j)=0$ for all $f \in L^{1}(G)$ and $j \in C_{0}(G) \cap I^{\perp}$. By the $w^{*}$-closedness of $I$ we may conclude that $f * \mu \in I$. On the other hand, if $g \in I$, then $\left(g_{x}, Q j_{x}\right)=0$ for all $j \in C_{0}(G)$ and $x \in G$; so $(g, R j)=0$ and $(g * \mu, j)=$ $(g, j)$ for all $j$. Therefore, $g=g * \mu$ for $g \in I$. Hence, $I=L^{1}(G) * \mu$. We also see that for all $f \in L^{1}(G)$ (take $g=f * \mu$ ), $f * \mu=f * \mu * \mu$. Consequently, $\mu$ is idempotent.

$(\beta) \Rightarrow(\gamma)$. Put $P f=f * \mu\left(f \in L^{1}(G)\right)$.

Remarks. Observe that the $w^{*}$-closedness was not used in proving the implication $(\alpha) \Rightarrow(\delta)$. In fact, by [4] and Part II below, $(\alpha)$ and $(\delta)$ are equivalent for all closed ideals in $L^{1}(G)$, in case $G$ is abelian.

If one applies the proof of $(\delta) \Rightarrow(\beta)$ to an $I$ that is nor $w^{*}$-closed, one finds an idempotent measure $\mu$ such that $L^{1}(G) * \mu$ lies in the $w^{*}$-closure of $I$, while $I$ is contained in the $w^{*}$-closed set $\left\{g \in L^{1}(G): g=g * \mu\right\}$. Thus, $L^{1}(G) * \mu=w^{*}-\mathrm{Cl}(I)$, the $w^{*}$-closure of $I$. Consequently, if $I$ is any closed left ideal in $L^{1}(G)$ such that $(\delta)$ holds, then $w^{*}-\mathrm{Cl}(I)$ has a right approximate identity.

From the proof of the implication $(\beta) \Rightarrow(\gamma)$ it follows that actually $P$ can be chosen so that

$$
P(f * g)=f * P g \quad\left(f, g \in L^{1}(G)\right) .
$$


One can derive $(\delta)$ from $(\gamma)$ by defining $Q$ by

$$
(f, Q b)=(f-P f, b) \quad\left(f \in L^{1}(G) ; b \in L^{\infty}(G)\right) .
$$

Thus, we can choose $Q$ so that

$$
Q(f * b)=f * Q b \quad\left(f \in L^{1} \cdot(G) ; b \in L^{\infty}(G)\right) .
$$

Part II. Bounded approximate identities in ideals of commutative group algebras. In this part, $G$ is a locally compact abelian group, $\Gamma$ its dual group. Since $G$ has the property $\left(P_{1}\right)$ it follows from Theorem 2 that if $I$ is a closed ideal in $L^{1}(G)$ and if there exists a continuous projection of $L^{1}(G)$ onto $I$, then $I$ has a bounded approximate identity. It is as yet unknown whether the converse holds. The first few theorems of this part serve, partly as a preparation for our Ma in Theorem, partly as an illustration for the close parallel between continuous projections and bounded approximate identities.

Theorem 5. Let $X, Y \subset \Gamma$ be closed.

(i) If $k X$ and $k Y$ bave bounded approximate identities, then so does $k(X \cup Y)$.

(ii) If there exist continuous projections $P_{X}$ of $L^{1}(G)$ onto $k X$ and $P_{Y}$ of $L^{1}(G)$ onto $k Y$, then there exists a continuous projection $P$ of $L^{1}(G)$ onto $k(X \cup Y)$ provided that there exist continuous linear maps $S: k X \rightarrow k(X \cup Y)$ and $T: k Y \rightarrow k(X \cup Y)$ such that $S+T=I$ on $k(X \cup Y)$. This condition is satisfied if there is a $\mu \in M(G)$ such that $\hat{\mu}=1$ on $X \backslash Y, \hat{\mu}=0$ on $Y \backslash X$.

Proof. (i) Let $\left(u_{i}\right),\left(v_{j}\right)$ be bounded approximate identities in $k X$ and $k Y$ respectively. If $f_{1}, \cdots, f_{n} \in k(X \cup Y)$ and $\epsilon>0$, there is a $u_{i}$ such that $\left\|f_{k} * u_{i}-f_{k}\right\| \leq 1 / 2 \epsilon$ for each $k$, and there is a $v_{j}$ such that $\left\|\left(f_{k} * u_{i}\right) * v_{j}-\left(f_{k} * u_{i}\right)\right\| \leq$ $1 / 2 \epsilon$ for each $k$. Then $u_{i} * v_{j} \in k(X \cup Y)$ while $\left\|f_{k} *\left(u_{i} * v_{j}\right)-f_{k}\right\| \leq \epsilon$ for each $k$.

(ii) Put $P=S P_{X}+T P_{Y}$; then $P: L^{1}(G) \rightarrow k(X \cup Y)$ and $P=I$ on $k(X \cup Y)$. If $\mu \in M(G), \hat{\mu}=1$ on $X \backslash Y$ and $\hat{\mu}=0$ on $Y \backslash X$, then we can define $S, T$ by

$$
\begin{array}{ll}
S f=\mu * f & (f \in k X), \\
T f=f-\mu * f & (f \in k Y) .
\end{array}
$$

Corollary 6. If there exists a continuous projection of $L^{1}(G)$ onto $k X$, and if $Y \subset \Gamma$ is finite, there exists a continuous projection of $L^{1}(G)$ onto $k(X \cup Y)$. (Trivially, there is a continuous projection of $L^{1}(G)$ onto $k Y$, as $k Y$ bas finite codimension.)

Corollary 7. Let $X, Y$ be disjoint, closed subsets of $\Gamma$ and assume that there exists a $\mu \in M(G)$ such that $\hat{\mu}=1$ on $X, \hat{\mu}=0$ on $Y$. (By [12, \$2.6.2] this is true if either $X$ or $Y$ is compact.)

(i) There is a bounded approximate identity in $k(X \cup Y)$ if and only if there exist bounded approximate identities in $k X$ and in $k Y$. 
(ii) There exists a continuous projection of $L^{1}(G)$ onto $k(X \cup Y)$ if and only if there exist continuous projections of $L^{1}(G)$ onto $k X$ and $k Y$.

Proof. In both cases the sufficiency has been proved above.

(i) Let $\left(u_{i}\right)$ be a bounded approximate identity in $k(X \cup Y)$ and $\left(v_{j}\right)$ a bounded approximate identity in $L^{1}(G)$. Then $\left(u_{i} * \mu+v_{j}-\mu * v_{j}\right)$ and $\left(u_{i}-u_{i} * \mu+\mu * v_{j}\right)$ are bounded approximate identities in $k X$ and $k Y$, respectively. (Note that $\mu * f \in k(X \cup Y)$ if $f \in k X$, and $f-\mu * f \in k(X \cup Y)$ if $f \in k Y$.)

(ii) Let $P$ be a continuous projection onto $k(X \cup Y)$. Define $Q: L^{1}(G) \rightarrow$ $L^{1}(G)$ by

$$
Q f=P(\mu * f)+f-\mu * f \quad\left(f \in L^{1}(G)\right) .
$$

Then $Q: L^{1}(G) \rightarrow k X$ and $Q=I$ on $k X$, so $Q$ is a projection onto $k X$. Similarly, $f \mapsto P(f-\mu * f)+\mu * f$ is a projection onto $k Y$.

At this stage the obvious question is whether Theorem 5 (ii) remains true if one drops the condition that the maps $S$ and $T$ exist. From Theorem 10 (i) it will become apparent that the existence of a bounded approximate identity in a closed ideal $I$ of $L^{1}(G)$ would imply the existence of a continuous projection from $L^{1}(G)$ onto $I$ if (and only if) the above question should be answered affirmatively.

The problem was raised first by $H$. P. Rosenthal who mentioned the following particular case. If $G=\mathbf{R}$, and $\alpha$ is an irrational real number, then there exist continuous projections of $L^{1}(G)$ onto $k \mathbf{Z}$ and onto $k(\alpha \mathbf{Z})$. (See [9] or Lemma 11 of this paper.) Does there exist a continuous projection onto $k(\mathbf{Z} \cup a \mathbf{Z})$ ? The authors have not been able to answer this question.

Theorem 8. Let $A$ be a closed subalgebra of $L^{1}(G)$. If $A \cap k(\{1\})$ bas a bounded approximate identity, so does $A$.

Proof. We may assume $A \not \subset k(\{1\})$. Take $b \in A ; \int b=1$. If $\left(u_{i}\right)$ is a bounded approximate identity for $A \cap k(\{1\})$, then $u_{i}-b * u_{i}+b$ is a bounded approximate identity for $A$ (observe that $f-f * b \in A \cap k(\{1\})$ for all $f \in A$ ).

Theorem 9. Let $A$ be a closed subalgebra of $L^{1}(G)$. If there is a continuous projection of $L^{1}(G)$ onto $A \cap k(\{1\})$, then there is a continuous projection of $L^{1}(G)$ onto $A$.

Proof. Let $P: L^{1}(G) \rightarrow A \cap k(\{1\})$ be a continuous projection. We may assume $A \not \subset k(\{1\})$. Choose $b \in A$ so that $P b=0$ and $\int b=1$. Then $f \mapsto P f+$ $\left(\int f\right) b$ is a continuous projection from $L^{1}(G)$ onto $A$.

For the sake of easy citation, we recapitulate a number of results concerning the structure of closed sets in the coset ring of $G_{d}$, i.e., $G$ with the discrete topology. From [6] we borrow the terms Calderón set and spectral set in preference to Rudin's C-set and S-set and to the terms Wiener-Ditkin set and Ditkin set, used 
by Reiter in [7]. Our Calderón sets are called Ditkin sets by Gilbert [4]. These results are essentially due to I. E. Gilbert (see [3] and also [13]).

Theorem 10. Let $G$ be an abelian topological group.

(i) If $a_{1} H_{1}, \cdots, a_{n} H_{n}$ are cosets of $G$, then there exists an open subgroup $H$ of $G$ such that int $\bigcup_{i}\left(a_{i} H_{i}\right)$ is a union of finitely many cosets of $H$.

(ii) Let $A$ be an element of the coset ring of $G_{d}$. Then $\bar{A}$ can be written as

$$
\bar{A}=\bigcup_{i=1}^{n} x_{i}\left(H_{i} \backslash F_{i} K_{i}\right),
$$

where, for each $i, x_{i}$ is an element of $G, H_{i}$ is a closed subgroup of $G, K_{i}$ is a relatively open subgroup of $H_{i}$ and $F_{i}$ is a finite subset of $H_{i}$. In particular, $\bar{A}$ lies in the coset ring of $G_{d}$.

(iii) Let $G, A, x_{i}, H_{i}, F_{i}$ and $K_{i}$ be as above and let $I=\left\{i: H_{i}\right.$ is open $\}$. Then

$$
\text { int } \bar{A}=\bigcup_{i \in I} x_{i}\left(H_{i} \backslash F_{i} K_{i}\right) \text {. }
$$

In particular, int $A$ belongs to the coset ring of $G$.

(iv) If every infinite closed subgroup of $G$ is open, then a subset $X$ of $G$ is a closed element of the coset ring of $G_{d}$ if and only if it is the union of a finite set and an element of the coset ring of $G$.

(v) If $G$ is locally compact with dual group $\Gamma$, then every closed element of the coset ring of $\Gamma_{d}$ is a Calderón set, hence a spectral set.

Now we turn to our main problem: describe the closed ideals in $L^{1}(G)$ that have bounded approximate identities. Our main tools are Theorem 2 and Theorem 10. The connection between them is the following lemma.

Lemma 11. Let $G$ be a locally compact abelian group, $\Gamma$ its dual group. Let $\Lambda$ be a closed subgroup of $\Gamma$ and $X$ an element of the coset ring of $\Lambda$. Then there exists a continuous projection of $L^{1}(G)$ onto $k X$.

Proof. Let $\Lambda_{\perp}=\{x \in G:(x, y)=1$ for every $\gamma \in \Lambda\}$. Let $G_{1}=G / \Lambda_{\perp}$, let $\Gamma_{1}$ be the dual group of $G_{1}$, and $\pi$ the natural map $G \rightarrow G_{1} \cdot \pi$ determines $\pi^{*}: \Gamma_{1} \rightarrow \Gamma$ by the formula $\pi^{*}\left(\gamma_{1}\right)=\gamma_{1} \circ \pi\left(\gamma_{1} \in \Gamma_{1}\right)$; this $\pi^{*}$ is a topological isomorphis $m$ $\Gamma_{1}$ onto $\Lambda$. By $[7, \$ 3.4 .4]$ there is a natural homomorphism $T$ of $L^{1}(G)$ onto $L^{1}\left(G_{1}\right)$ given by

$$
T f(\pi(x))=\int f(x y) d m(y)
$$

where $m$ denotes a Haar measure on $\Lambda_{\perp}$.

$T$ and $\pi^{*}$ are related by

$$
\left(\pi^{*} \gamma_{1}, f\right)=\left(\gamma_{1}, T f\right) \quad\left(\gamma_{1} \in \Gamma_{1} ; f \in L^{1}(G)\right) .
$$

There exists a linear isometry $S: L^{1}\left(G_{1}\right) \rightarrow L^{1}(G)$ such that $T S$ is the identity map $I_{1}$ of $L^{1}\left(G_{1}\right)$. (See [7, Chapter 8, \$2.7].) 
The set $X_{1}=\pi^{*-1} X$ is an element of the coset ring of $\Gamma_{1}$. By Cohen's Idempotent Measure Theorem [12, Chapter 31 there exists a $\mu_{1} \in M\left(G_{1}\right)$ such that $\hat{\mu}_{1}=\chi_{X_{1}}$. Then $P_{1}: f_{1} \mapsto \mu_{1} * f_{1}$ is a continuous projection of $L^{1}\left(G_{1}\right)$ onto $k X_{1}$. Now $S\left(I_{1}-P_{1}\right) T$ is a continuous linear map of $L^{1}(G)$ into $L^{1}(G)$; it is idempotent because $T S=I_{1}$. Hence, $P=I-S\left(I_{1}-P_{1}\right) T$ (where $I$ is the identity map $L^{1}(G) \rightarrow L^{1}(G)$ ) is a continuous projection of $L^{1}(G)$ into $L^{1}(G)$; its range is $\operatorname{Ker} S\left(I_{1}-P_{1}\right) T=$ $\operatorname{Ker}\left(I_{1}-P_{1}\right) T=T^{-1}\left(\operatorname{Im} P_{1}\right)=T^{-1}\left(k X_{1}\right)=k X$.

In H. Reiter's book [7], a commutative Banach algebra $A$ is said to have a bounded approximate identity if the re exists a number $c>0$ such that for every $a \in A$ and $\epsilon>0$ there is a $u \in A,\|u\| \leq c$, for which $\|a-u a\| \leq \epsilon$. We shall see that for closed ideals in $L^{1}(G)$ the presence of a bounded approximate identity in Reiter's sense is equivalent to that of a bounded approximate identity as we defined it before Theorem 2 of this paper.

Lemma 12. Let $A$ be a commutative Banach algebra. Assume that for every $a \in A$ there exists a bounded sequence $\left(u_{n}\right)$ such that $\lim u_{n} a=a$. Then $A$ bas a bounded approximate identity in the sense of Reiter [7], i.e., there exists a $c>0$ sucb that $a \in \mathrm{Cl}\{x a:\|x\| \leq c\}$ for all $a \in A$. (The converse is trivial.)

Proof. For $m \in \mathbf{N}$ let

$$
A_{m}=\{a \in A: a \in \mathrm{Cl}\{x a:\|x\| \leq m\}\} .
$$

It is easy to see that $A_{m}$ is a closed subset of $A$. By the given condition on $A$, $\bigcup_{A_{m}}=A$. By the Baire Category Theorem one of the $A_{m}$, say $A_{m_{0}}$, contains a nonempty open ball $B$. Then $\mathrm{C} B-\mathrm{CB}$ is a linear subspace of $A$ with nonempty interior, so $\mathrm{C} B-\mathrm{C} B=A$. Any $a \in A$ can be written as $a=a_{1}-a_{2}$ where $a_{1}, a_{2}$ $\epsilon \mathrm{C} B \subset A_{m_{0}}$. For any $\epsilon>0$ there exist $x_{1}, x_{2}$ such that $\left\|x_{i}\right\| \leq m_{0}$ and $\left\|a_{i}-x_{i} a_{i}\right\| \leq 1 / 2\left(1+m_{0}\right)^{-1} \epsilon$ for $i=1$, 2. Putting $x=x_{1}+x_{2}-x_{1} x_{2}$ we obta in $\|x\| \leq 2 m_{0}+m_{0}^{2}$ and $\|a-x a\| \leq \epsilon$. Thus, we may take $c=2 m_{0}+m_{0}^{2}$.

Now we turn to our main theorem.

Theorem 13. Let $G$ be a locally compact abelian group with dual group $\Gamma$, $l$ a closed ideal in $L^{1}(G)$. The following conditions are equivalent.

(a) I has a bounded approximate identity.

( $\beta$ ) I is factorable, i.e., there exists a $c>0$ such that for every $f \in I$ and $\epsilon>0$ we can write $f=g_{1} * g_{2}$ where $g_{1}, g_{2} \in I,\left\|g_{1}\right\| \leq c$, and $\left\|f-g_{2}\right\| \leq \epsilon$.

$(\gamma)$ For every $f \in I$ there is a bounded sequence $\left(u_{n}\right)$ in I sucb that $\lim u_{n} * f=f$.

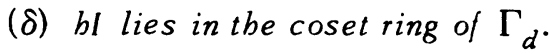

(є) $I=k X$ for some $\Gamma$-closed element $X$ of the coset ring of $\Gamma_{d}$.

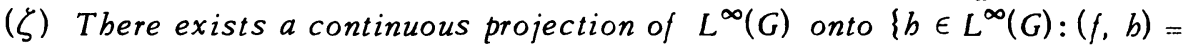
0 for all $f \in I\}$. 
Proof. $(\alpha) \Rightarrow(\beta)$ is a special case of P. Cohen's Factorization Theorem [2].

$(\beta) \Rightarrow(\gamma)$. If $c, f, \epsilon, g_{1}, g_{2}$ are as in $(\beta)$, then $\left\|g_{1} * f-f\right\|=\left\|g_{1} *\left(f-g_{2}\right)\right\| \leq c \epsilon$.

$(\gamma) \Rightarrow(\delta)$. By Lemma 12 there exists a $c>0$ such that for every $\epsilon>0$ and $f \in I$ we can find $u \in I$ for which $\|u\| \leq c$ and $\|u * f-f\| \leq \epsilon$.

Let $I_{0}=\left\{f \in L^{1}(G):\right.$ Supp $\hat{f}$ is compact; $\left.b I \cap \operatorname{Supp} \hat{f}=\varnothing\right\}$. By $[12, \S 7.2 .5]$ $I_{0} \subset I$. Let $f_{1}, \cdots, f_{n} \in I_{0}$ and $\epsilon>0$. There exists an $f \in I_{0}$ such that $\hat{f}=1$ on Supp $\hat{f}_{i}(i=1, \cdots, n)[12, \$ 2.6 .2]$. Then for each $i$ we have $\hat{f} \hat{f}_{i}=\hat{f}_{i}$, so $f * f_{i}=f_{i}$. As we have seen, there is a $u \in I,\|u\| \leq c$, for which $\|u * f-f\| \leq \epsilon\left(\max \left\|f_{i}\right\|\right)^{-1}$. Then for each $i$ we have $\left\|u * f_{i}-f_{i}\right\|=\left\|(u * f-f) * f_{i}\right\| \leq \epsilon$.

It follows from these considerations that $I$ contains a bounded net $\left(u_{j}\right)$ such that $\lim u_{j} * f=f$ for every $f \in I_{0}$. Let $\bar{G}$ be the Bohr compactification of $G$, and $\bar{\Gamma}$ its dual group. Every continuous almost periodic function $f$ on $G$ induces a continuous function $\bar{f}$ on $\bar{G}$. In particular, every $\gamma \in \Gamma$ induces $\bar{\gamma} \in \bar{\Gamma}$, and the map $\gamma \mapsto \bar{\gamma}$ is a surjective group isomorphism. The map $f \mapsto \bar{f}$ induces a continuous linear map $\Omega: L^{1}(G) \rightarrow M(\bar{G})$. For every $f \in L^{1}(G)$ and $\gamma \in \Gamma,(\gamma, f)=$ $(\bar{\gamma}, \underline{\Omega} f)$. The net $\left(\Omega u_{j}\right)$ is norm-bounded, hence has a $w^{*}$-limit point $\mu \in M(\bar{G})$. We may assume $w^{*} \lim \Omega u_{j}=\mu$. In particular, for every $\gamma \in \Gamma$ we have $\lim \left(\gamma, u_{j}\right)=$ $(\bar{\gamma}, \mu)$. As $u_{j} \in I$ it follows that $(\bar{\gamma}, \mu)=0$ for $\gamma \in b I$. But if $\gamma \in \Gamma$ and $\gamma \notin b I$, we can choose $f \in I_{0},(\gamma, f)=1$; then $(\bar{\gamma}, \mu)=\lim \left(\gamma, u_{j}\right)(\gamma, f)=\lim \left(\gamma, u_{j} * f\right)=$ $(\gamma, f)=1$. Thus, $\hat{\mu}$ is the characteristic function of $\{\bar{\gamma}: \gamma \in \Gamma \backslash b I\}$. By Cohen's Theorem on Idempotent Measures we conclude that $\{\bar{\gamma}: \gamma \in b l\}$ lies in the coset ring of $\bar{\Gamma}$, so that $b l$ lies in the coset ring of $\Gamma_{d}$.

$(\delta) \Rightarrow(\epsilon)$. By Theorem $10(\mathrm{v}), b I$ is a spectral set, so $I=k b I$.

$(\epsilon) \Rightarrow(\alpha)$. Applying Lemma 11 and Theorem 10 (ii), we see that $X$ can be written as a finite union $X=\gamma_{1} X_{1} \cup \ldots \cup \gamma_{n} X_{n}$ where $\gamma_{i} \in \Gamma$ and $X_{i}$ is such that there exists a continuous projection of $L^{1}(G)$ onto $k X_{i}$. The map $f \mapsto \gamma_{i}^{-1} f$ is a linear isometry of $L^{1}(G)$ onto $L^{1}(G)$ that maps $k X_{i}$ onto $k\left(\gamma_{i} X_{i}\right)$; so there exist continuous projections of $L^{1}(G)$ onto the $k\left(\gamma_{i} X_{i}\right)$. Consequently, by Theorem 2 each $k\left(\gamma_{i} X_{i}\right)$ has a bounded approximate identity. Now use Theorem 5 (i).

$(\epsilon) \Leftrightarrow(\zeta)$. See [4].

Remark. The equivalence of $(\alpha)$ and $(\delta)$ was studied in $[13,2.8]$.

The following consequence of the above theorem is curious.

Corollary 14. If $\Gamma$ is a locally compact abelian group, if $X$ is a closed element of the coset ring of $\Gamma_{d}$, and if $Z$ is a compact, relatively open subset of $X$, then $Z$ lies in the coset ring of $\Gamma_{d}$.

One could apply the technique of the proof of the implication $(\gamma) \Rightarrow(\delta)$ in a slightly different way. View $L^{1}(G)$ as a subspace of $M(G)$. The net $\left(u_{i}\right)$ has a $w^{*}$-limit point $\mu \in M(G)$. It is not difficult to prove that $\mu$ is an idempotent 
measure whose Fourier-Stieltjes transform is just the characteristic function of $\Gamma \backslash$ int $b I$. (See Theorem 10(iii).)

The conditions $(\alpha)-(\zeta)$ are not implied by the existence of a (possibly unbounded) approximate identity in $I$. As an example, let $\Gamma$ be discrete. Then every $X \subset \Gamma$ is Calderón $[6,39.39$ (b)], so every $k X$ has an approximate identity.

Suppose that every inf inite closed subgroup of $\Gamma$ is open. Every closed element $X$ of the coset ring of $\Gamma_{d}$ then is the union of a finite set $\Phi$ and a set $Y$ that can be written as

$$
Y=\bigcup_{i=1}^{n} \gamma_{i}\left(\Lambda_{i} \backslash \Phi_{i} \Delta_{i}\right)
$$

where $\gamma_{i} \in \Gamma, \Lambda_{i}$ is an open subgroup of $\Gamma, \Delta_{i}$ is an open subgroup of $\Lambda_{i}$, and $\Phi_{i} \subset \Gamma$ is finite. Then $Y$ lies in the coset ring of $\Gamma$, and by the Idempotent Measure Theorem there is an idempotent $\mu \in M(G)$ whose Fourier-Stielties transform is the characteristic function of $\Gamma \backslash Y$ 。 Then $f \mapsto f * \mu$ is a continuous projection of $L^{1}(G)$ onto $k Y$.

Applying Corollary 6, Theorem 10(iv) and Lemma 11 we obtain

Corollary 15. Let $G, \Gamma, I$ be as in Theorem 13. Assume that every infinite closed subgroup of $\Gamma$ is open. Then the conditions $(\alpha)-(\zeta)$ are equivalent to

$(\eta)$ bI is the union of a finite set and an element of the coset ring of $\Gamma$.

(v) There exists a continuous projection of $L^{1}(G)$ onto $I$.

Added in proof. Most of Theorem 13 was proved independently by H. Reiter who published it as Theorem 2 in Chapter 17 of his book $L^{1}$-algebras and Segal algebras, Lecture Notes in Math., vol. 231, Springer-Verlag, Berlin and New York, 1971.

It was proved by M. Altman (Contracteurs dans les algèbres de Banach, C. R. Acad. Sci. Paris Sér. A 274 (1972), A399-A400) that for any Banach algebra the existence of a bounded left approximate identity in Reiter's sense is equivalent to the existence of a bounded left approximate identity as the term is used in this paper.

\section{REFERENCES}

1. N. Bourbaki, Eléments de mathématique. XXV. Part. I. Livre VI: Intégration. Chap. 6: Intégration vectorielle, Actualités Sci. Indust., no. 1281, Hermann, Paris, 1959. MR 23 \#A2033.

2. P. J. Cohen, Factorization in group algebras, Duke Math. J. 26 (1959), 199-205. MR $21 \# 3729$.

3. J. E. Gilbert, On a strong form of spectral synthesis, Ark. Mat. 7 (1969), 571575. MR $43 \# 3740$.

4. - On projections of $L^{\infty}(G)$ onto translation-invariant subspaces, Proc. London Math. Soc. (3) 19 (1969), 69-88. MR $39 \# 6019$. 
5. F. P. Greenleaf, Invariant means on topological groups and their applications, Van Nostrand Reinhold, New York, 1969. MR 40 \#476.

6. E. Hewitt and K. A. Ross, Abstract harmonic analysis. Vols. 1, 2, Die Grundlehren der math. Wissenschaften, Bände 115, 152, Springer-Verlag, Berlin and New York, 1963, 1970. MR 28 \#158; MR 41 \#7378.

7. H. Reiter, Classical harmonic analysis and locally compact groups, Oxford Univ. Press, Oxford, 1968.

8. _-_, Sur certains idéaux dans $L^{1}(G)$, C. R. Acad. Sci. Paris Sér. A-B 267 (1968), A882-A885. MR 39 \#6025.

9. H. P. Rosenthal, Projections onto translation-invariant subspaces of $L^{p}(G)$, Mem. Amer. Math. Soc. No. 63 (1966), 84 pp. MR 35 \#2080.

10. - On the existence of approximate identities in ideals of group algebras, Ark. Mat. 7 (1967), 185-191. MR 39 \#719.

11. W. Rudin, Projections on invariant subspaces, Proc. Amer. Math. Soc. 13 (1962), 429-432. MR 25 \#1460.

12. - Fourier analysis on groups, Interscience Tracts in Pure and Appl. Math., no. 12, Interscience, New York, 1962. MR 27 \#2808.

13. B. M. Schreiber, On the coset ring and strong Ditkin sets, Pacific J. Math. 32 (1970), 805-812. MR 41 \#4140.

DEPARTMENT OF MATHEMATICS AND STATISTICS, UNIVERSITY OF MASSACHUSETTS, AMHERST, MASSACHUSETTS 01002 (Current address of Teng-sun Liu and Ju-kwei Wang)

DEPARTMENT OF MATHEMATICS, CATHOLIC UNIVERSITY, NIJMEGEN, THE NETHERLANDS (Current address of Arnoud van Rooij) 